\title{
The spectrum of medical errors: when patients sue
}

This article was published in the following Dove Press journal:

International Journal of General Medicine

20 July 2012

Number of times this article has been viewed

\author{
Barry D Kels' \\ Jane M Grant-Kels² \\ 'Department of Surgery, \\ ${ }^{2}$ Department of Dermatology, \\ University of Connecticut Health \\ Center, Farmington, CT, USA
}

Correspondence: Jane M Grant-Kels Department of Dermatology, University of Connecticut Health Center,

263 Farmington Avenue, MC 6230,

Farmington, CT 06030, USA

$\mathrm{Tel}+\mathrm{I} 8606793474$

Fax + I 860679 |267

Email grant@uchc.edu
Abstract: Inarguably medical errors constitute a serious, dangerous, and expensive problem for the twenty-first-century US health care system. This review examines the incidence, nature, and complexity of alleged medical negligence and medical malpractice. The authors hope this will constitute a road map to medical providers so that they can better understand the present climate and hopefully avoid the "Scylla and Charybdis" of medical errors and medical malpractice. Despite some documented success in reducing medical errors, adverse events and medical errors continue to represent an indelible stain upon the practice, reputation, and success of the US health care industry. In that regard, what may be required to successfully attack the unacceptably high severity and volume of medical errors is a locally directed and organized initiative sponsored by individual health care organizations that is coordinated, supported, and guided by state and federal governmental and nongovernmental agencies.

Keywords: primum non nocere, medical negligence, medical malpractice, Six Sigma

\section{Introduction}

The etiology of the universal medical caveat "primum non nocere" 1 translated as "first, do no harm" - is most often attributed to Hippocrates, although evidence exists that he may not have employed that precise phraseology. However, regardless of its origins, the caveat remains the foundation of ethical, successful, and skillful medical practice. Therefore, great concern has developed both within and outside the medical profession over the last 2 decades in response to a respected body of evidence averring that medical errors represent a serious, injurious, and expensive problem plaguing the entirety of the US health care system. Furthermore, the increased awareness and frequency of medical malpractice litigation has created an ominous environment that simultaneously diminishes and jeopardizes the sanctity of the physician-patient relationship.

\section{Statistics showing medical errors are a profound problem vis-à-vis health care costs and quality of care}

Harvard's 1991 Medical Practice Study asserted that in the Orwellian year of 1984, adverse events transpired in approximately 37 out of every 1000 admissions to New York State hospitals. ${ }^{2}$ The study retrospectively examined more than 30,000 New York State hospital admissions. Within that study, adverse events were defined as injuries resulting from omissions or commissions related to medical 
management, as opposed to the underlying diseases or medical conditions of individual patients. ${ }^{2}$ In this context, adverse events may be caused by mistakes or negligence constituting incorrect, inadequate, or substandard treatment of a patient by a health care professional. Significantly, more than $2 \%$ of the aforementioned adverse events proximately caused permanent disability, while more than $13 \%$ of the documented adverse events proximately caused the death of affected patients. ${ }^{3}$

In the year 2000, approximately a decade after Harvard's 1991 Medical Practice Study, the Institute of Medicine (IOM) published its landmark study To Err is Human: Building a Safer Health System. ${ }^{4}$ Within the IOM treatise, the authors extrapolated from the Harvard 1991 Medical Practice Study as well as companion studies conducted in Colorado and $\mathrm{Utah}^{5}$ and estimated that somewhere between 44,000 and 98,000 hospital deaths transpire nationally per annum because of medical errors. Furthermore, that same IOM report postulated that medical errors in the 50 states were responsible for \$ US17-\$29 billion in avoidable annual excess expenditures. ${ }^{4}$ Finally, this report raised the specter of Americans delaying and/or avoiding necessary health care intervention because of the knowledge and/or fear of being subjected to widespread medical errors. ${ }^{4}$

Shortly thereafter, in 2001, the IOM published another damning study, entitled Crossing the Quality Chasm: A New Health System for the 21st Century. ${ }^{6}$ This report found health care within the 50 states to be substandard in multiple dimensions. Finally, 2 years later, McGlynn et $\mathrm{al}^{7}$ published a study in the New England Journal of Medicine wherein it was demonstrated that adults admitted to US hospitals were provided only slightly more than $50 \%$ of accepted optimal treatment.

Although the statistical validity of extrapolating from the health care statistics in a single or even several individual states to the global quality of US medical care is open to some dispute, according to these aforementioned rather condemnatory studies there can be little doubt that medical omissions and commissions represent a profound problem regarding the quality, cost, and success of US medical care.

\section{Current terminology regarding medical errors}

In an effort to promote a mutual understanding of the essential nature of medical errors, the Quality Interagency Coordination Task Force has modified the IOM's definition of a medical error to include the following: "the failure of a planned action to be completed as intended or the use of a wrong plan to achieve an aim. Errors can include problems in practice, products, procedures, and systems." 8

Sophisticated participants and other stakeholders in the twenty-first-century US health care system understand that adverse outcomes are not necessarily associated with the existence of medical error(s). Patients may respond poorly to treatment despite receiving the highest quality medical care. Moreover, an adverse response to medical care may not necessarily imply the intervention of medical error. Adverse events are broadly defined as injuries related to medical treatment resulting in measurable disability. ${ }^{9}$ In fact, an unpreventable adverse event is specifically defined as a "complication that cannot be prevented given the current state of medical knowledge." ${ }^{8}$ Conversely, medical errors include adverse events that are, in fact, preventable considering the current state of medical knowledge. ${ }^{8}$ In the interest of thoroughness, the definition of medical error may be expanded from mere preventable adverse events proximately causing actual harm to include near misses, which are best understood as situations or events that could have resulted in accident, injury, or illness but were averted by providence or timely intervention. ${ }^{8}$

It may similarly be appropriate to consider medical errors within the larger context of US health care quality where concerns are generally agreed to encompass three broad categories of arguably inappropriate utilization: (1) overuse (ie, the specific intervention is unlikely to demonstrate affirmative benefit), (2) underuse (ie, an arguably beneficial intervention is not employed), and (3) misuse (ie, an intervention is inappropriately undertaken). ${ }^{10}$ For the sake of this discussion, the authors shall only address medical errors as mistakes in practice or thought, without addressing the specific rubrics of overuse, underuse, and misuse; albeit, the vast majority of medical errors almost certainly fall within the latter classification of misuse.

\section{Procedures in place regarding medical errors}

Over the last 25 years, numerous initiatives have been contemplated, suggested, and implemented in attempts to adapt Six Sigma quality analysis to the reduction and prevention of medical errors. Six Sigma refers to the Greek letter that represents standard deviation from a normally distributed curve. ${ }^{10}$ More specifically, adherence to Six Sigma quality assurance seeks performance exceeding six standard deviations better than the norm such that only 3.4 errors per million are deemed acceptable and the error-free rate exceeds $99.99966 \% .{ }^{11} \mathrm{Six}$ Sigma quality analysis has been credited with dramatically 
reducing errors and customer service problems at the Federal Aviation Administration and industrial leviathans such as Motorola and General Electric.

Application of Six Sigma solutions at non-health care industries has been characterized by the following systematic cultural initiatives:

1. Intolerance of high mistake rates coupled with bold targets for fault diminution enterprises;

2. The development of tracking mechanisms to expose mistakes;

3. Reliance upon copious reports of errors and "near misses";

4. The methodical investigation of errors including formal root cause analyses;

5. A systematic approach to error reduction including an expansive array of human and technical factors and potential organizational remedies;

6. A focus upon systems improvements detached from the identification of individual culpability;

7. An alteration in the organizational culture to enhance safety and error reduction;

8. The allocation of sufficient resources to enable error prevention programs and expand the knowledge base;

9. The expansion of the culture to identify and implement original solutions. ${ }^{8}$

\section{Comparison of the US health care industry with non-health care industries}

It is well documented that the error rate is considerably higher in the US health care industry (HCI) than in other industries. ${ }^{12}$ In a single study of intensive care units, it was demonstrated that the correct action was implemented 99\% of the time. Despite that seemingly encouraging statistic, a 99\% success rate translated into 1.7 daily cognizable medical errors. Significantly, if that error rate were reduced by a factor of ten to a $99.9 \%$ success rate, in the banking industry it would still translate into 32,000 checks per hour deducted from the wrong account by the US banking industry. This would obviously represent an unacceptable and untenable error rate. ${ }^{9}$

It is undeniable that the HCI shares substantive characteristics with industries such as aviation and banking. Specifically, all three industries depend upon systems wherein humans and technology interact to achieve a defined outcome. However, it is virtually inarguable that the HCI is infinitely more complex than complex non-HCIs such as aviation and banking. For example, it has been demonstrated that an intensive care patient is the recipient of an average of 178 different manipulations per day involving the interaction of treatment, monitoring, and support systems. ${ }^{9}$ Therefore, numerous difficulties exist in any attempt to adapt industrial Six Sigma quality initiatives to the HCI. Specifically, the balkanized organization and fragmented nature of the US HCI arguably enhance the potential for errors and make the $\mathrm{HCI}$ idiosyncratically resistant to centralized attempts to diminish errors with the intent of augmenting patient safety.

The extant organizational nature of the US HCI similarly makes the industry unreceptive to quality improvement initiatives. More pointedly, US health care providers are often isolated and detached from their colleagues, and interaction and communication among specialties is commonly suboptimal. Information regarding mutually shared patients is often delayed or unavailable. Initiatives to enhance patient safety are often incorporated into one specialty absent extension to colleagues in primary care and other specialties. Furthermore, the individual variation in clinical response of vast numbers of patients similarly makes the HCI unreceptive to safety initiatives insofar as it can be difficult to identify and categorize errors. Moreover, the very fact that a patient is seriously ill may serve to camouflage discernible errors by attributing an adverse reaction to the individual patient's underlying disease(s) rather than the prescribed treatment. In addition, errors may be masked by both the biologic variability of a large patient population in response to a specific treatment and the failure to associate an adverse reaction with a particular agent or procedure. In as much as medical errors usually affect a single organism at a time, it may be difficult to appropriately associate a cluster of adverse outcomes with a particular inciting treatment. Finally, the fear of litigation, retribution, and abrogation of patient privacy almost certainly contributes to the underreporting of identifiable medical errors.

\section{Current programs to prevent medical errors Local programs}

Since the turn of the twenty-first century, patients, regulators, purchasers, and accreditation agencies have demanded an enhanced focus upon health care quality. Most hospitals and health plans collect and monitor data regarding specific events (eg, falls) and/or outcomes (eg, anesthesia deaths) ${ }^{8}$ Hospitals generally use these data for performance improvement and continuous quality improvement initiatives. Determinations regarding what parameters to investigate commonly are based upon multiple factors including, but not necessarily limited 
to, the type, seriousness, and severity of identified issues at the institutional level; the difficulty of accumulating data and initiating an identifiable improvement initiative; and related issues such as potential cost savings and accreditation requirements. ${ }^{8}$ Such initiatives are much less common outside of the acute care hospital setting. Infection prevention and control programs represent the bellwether for such programs wherein well-designed and well-implemented programs have been demonstrated to dramatically reduce nosocomial infections. As such, infection control is a substantive part of most hospital accreditation initiatives.

\section{Regional and national programs}

Several national and regional programs exist to encourage, monitor, and organize reporting and amelioration of specific health care adverse events and errors. Among the more prominent programs on a national level, the US Food and Drug Administration ${ }^{13,14}$ oversees mandatory reporting programs regarding drug, device, vaccine, and blood product matters, while the Centers for Disease Control's National Nosocomial Infections Surveillance System ${ }^{15}$ monitors health care-related infections. Furthermore, various state agencies - including those in the states of New York, Massachusetts, and Florida - monitor various targeted health parameters. ${ }^{16}$ Finally, nongovernmental organizations such as the Joint Commission on Accreditation of Healthcare Organizations oversee medical error reporting programs. ${ }^{8}$

Interestingly, the vast majority of reporting agencies have minimal, if any, enforcement power to insure thorough and accurate reporting. These agencies rely almost entirely upon the voluntary reporting system. In point of fact, most observers believe that significant underreporting of adverse events and medical errors is commonplace for various reasons including fear of retribution and litigation as well as the failure of reporters to receive actionable follow-up information from the recipient agency.

\section{Successful medical error reporting programs}

Despite the discouraging anecdotal reports and IOM statistics regarding the frequency and severity of medical errors within the US HCI, several successful initiatives have been launched and have proved invaluable in reducing the frequency and severity of medical errors. Most prominently, the specialty of anesthesiology proved successful in reducing its error rate dramatically by first initiating a systematic analysis of errors. Subsequently through cooperation, technological improvements, standardization, simplification, and the adoption of practice guidelines, the specialty of anesthesia was able to reduce its error rate from 25-50 per million patients to something of the order of 4-7 per million patients. ${ }^{17}$ Similarly, the US Department of Veterans Affairs has reduced medication errors by $70 \%$ subsequent to the introduction of a handheld, wireless medication bar coding system. ${ }^{18}$

\section{Case studies}

\section{Case I: metastatic squamous cell carcinoma - squamous cell carcinoma and alleged abandonment}

A Caucasian sun-damaged patient in his early 60 s was a long-term patient of a well-respected community dermatologist. A shave biopsy of a crusted papule from the patient's temple was submitted to the university dermatopathology laboratory and signed out as "fragments of an actinic keratosis" with a note that the "pathology extended to the margins of the fragmented specimen" and that "a squamous cell carcinoma [SCC] could not unequivocally be ruled out". A deeper biopsy and further therapy was recommended. The dermatologist's office telephoned the patient and arranged for him to return for an excision 3 weeks later. When the patient returned to the dermatologist's office, the lesion had tripled in size.

The dermatologist immediately referred the patient to an independently practicing plastic surgeon who arranged to see the patient that same day. The referring dermatologist had requested that the plastic surgeon excise this lesion when they chatted on the telephone. The dermatologist assumed that the care of this problematic lesion had now been successfully transferred to the care of another physician. However, the plastic surgeon independently decided that this lesion was behaving more like a keratoacanthoma than a SCC. Since many keratoacanthomas can resolve spontaneously, the plastic surgeon made the regrettable decision to observe the lesion and deferred the surgical excision. The lesion did not resolve but stabilized in size and persisted. Because of various conflicts in the patient's and the surgeon's schedules, excisional surgery was postponed for 6 months. The excision at that later date revealed a SCC that invaded deeply into the dermis and subcutaneous tissue, with evidence of atypical squamous cells within vessel walls.

The patient went on to develop metastatic SCC that ultimately resulted in his untimely and arguably "wrongful" death. Both the plastic surgeon and the dermatologist were sued for medical malpractice. The plastic surgeon settled 
for the \$1 million limit of his malpractice insurance policy. The dermatologist did not settle in as much as he was of the opinion that he had not done anything improper. He lost the suit based upon the theory of abandonment of the patient. The jury opined that when the dermatologist received a letter from the plastic surgeon detailing the plastic surgeon's plans to observe the lesion and only perform an excision if the lesion did not regress, the dermatologist should have called the plastic surgeon or referred the patient to another plastic surgeon. The dermatologist lost the case beyond the $\$ 3$ million limit of his malpractice coverage, although ultimately the plaintiff's decedent settled for the maximum of the dermatologist's $\$ 3$ million policy limit. The next case study examines the theory of vicarious liability from another perspective.

\section{Case 2: pseudotumor cerebri - alleged vicarious liability}

A 32-year-old obese black female presented to a general ophthalmologist complaining of mild, bilateral diminution in vision and headaches transpiring over the preceding 6 weeks. Ophthalmologic examination revealed a best-corrected distance visual acuity of 20/25 in the right eye and 20/30 in the left eye. External examination and slit lamp biomicroscopy proved relatively unremarkable. Intraocular pressures were recorded as $16.0 \mathrm{mmHg}$ in both eyes. Gonioscopy revealed widely open anterior chamber drainage angles bilaterally. Humphrey automated perimetry revealed focal visual field defects with no diagnostic pattern. Optical coherence tomography was nondiagnostic. Finally, dilated examination of the ocular media and fundi revealed presumptive, bilateral papilledema.

The general ophthalmologist's provisional diagnosis was pseudotumor cerebri (PTC). To both confirm the diagnosis and facilitate appropriate referral to a neurologist/neuroophthalmologist, the general ophthalmologist arranged for the patient to be evaluated by a retinologist that same day. The retinologist confirmed the general ophthalmologist's clinical findings as well as the presumptive diagnosis of PTC. The retinologist specifically and voluntarily agreed to both accept responsibility for the ongoing care of the patient and to insure that the patient was further referred to a neurologist/neuro-ophthalmologist for definitive medical and/or surgical therapy. Moreover, the retinologist documented his acceptance of responsibility for the patient in a consultation summary letter. Subsequently, the retinologist did arrange neurologic consultation for the patient, who was then sent for magnetic resonance imaging prior to planned lumbar puncture. Unfortunately, the magnetic resonance imaging unit was nonfunctional when the patient arrived and the neurologist did not reschedule the study.

The patient was then lost to follow-up for approximately 1 weeks time, at which time she reappeared at the original general ophthalmologist's office complaining of some additional diminution in vision. The patient was then directly referred to a neuro-ophthalmologist who performed bilateral optic nerve fenestrations, albeit not before the patient developed bilateral apoplectic events resulting in best-corrected $20 / 400$ vision in the right eye and count fingers vision in the left eye. The patient sued the neurologist, retinologist, and general ophthalmologist. On advice of his insurer and counsel, the general ophthalmologist settled the suit for $\$ 225,000$. The retinologist settled the suit for $\$ 550,000$ and the neurologist settled for $\$ 1,500,000$.

Although a rather compelling case could be made that the retinologist and, particularly, the general ophthalmologist operated within the standard of care, the severe visual disability of the plaintiff created a situation in which the insurers and defense counsel were concerned about a potentially large jury award to a poor, minority, injured, and severely incapacitated plaintiff. This case again illustrates that it is exceedingly difficult for a provider to detach him- or herself from an established physician-patient relationship. Furthermore, this case of PTC similarly illustrates that health care providers may be subjected to vicarious liability for the acts and/or omissions of their consulting colleagues. In this case, all the physicians caring for the patient were in separate private practices and only related by being on staff at the same inner-city hospital. Vicarious liability in this scenario is represented by the fact that although the ophthalmologist and retinologist performed their jobs with expertise, they were held legally responsible for the action or lack thereof of the neurologist. Specifically, the final case study examines the theory of the borrowed servant doctrine (BSD) that is often used in institutional settings to impute liability to actors such as operating surgeons.

\section{Case 3: resident/supervisee error - the BSD}

A 62-year-old white male underwent thoracotomy. During the procedure, the attending surgeon asked the resident surgeon to identify landmarks $\mathrm{A}$ and $\mathrm{C}$ and to cut tissue at landmark B, midway between the two points. The resident surgeon correctly identified points $\mathrm{A}$ and $\mathrm{C}$ and acknowledged his intention to cut tissue midway between those two points. The attending surgeon requested that the resident 
confirm the specified landmarks on two separate and subsequent inquiries. Satisfied that the resident surgeon understood his instructions, the attending surgeon instructed the resident surgeon to cut the tissue at point B. The resident instead cut at point $\mathrm{D}$ and transected the pulmonary artery. The patient exsanguinated and died.

At the medical malpractice trial against the defendant hospital, resident surgeon, and attending surgeon, the jury ultimately found the attending surgeon not to be negligent in his capacity as the attending surgeon or in his supervisory capacity as the instructor of the resident. However, the jury did find the resident surgeon to be negligent for transecting the improper tissue. Furthermore, under the BSD, the jury found the attending surgeon liable for the negligence of the resident surgeon in as much as under the BSD the attending surgeon was deemed to have exercised actual supervision and control of the resident surgeon at the time of the surgical negligence. Hence the jury imputed the resident's negligence to the attending surgeon. The attending surgeon was temporarily faced with an $\$ 11$ million judgment that was ultimately reduced to the $\$ 1$ million limit of his medical malpractice insurance policy.

This case demonstrates that every attending physician who instructs medical students, residents, and fellows runs the continuing risk of the attachment of vicarious liability for the negligence of their supervisees. Furthermore, under the $\mathrm{BSD}$, the dispositive inquiry devolves to an examination of whether the attending physician exerted actual supervision and control of the supervisee. If, in the opinion of the jury, the attending did exercise actual supervision and control of the student, resident, or fellow, the attending may be found liable for the supervisee's negligence.

\section{Case study conclusions}

These three case studies illustrate many problems with both the US HCI and the US medical malpractice tort system, which has arguably become a counterproductive force regarding the effective and efficient delivery of US medical care. Moreover, the medical malpractice system has become more of a patient compensation system and a financial punitive system than a purveyor of justice or a way to improve or advance medical care. On the one hand, several of the defendants in these case studies should arguably never have been subjected to medical malpractice litigation. Unfortunately, however, a basic tenet among medical malpractice plaintiffs' attorneys is to seek out as many "deep pockets" as possible, regardless of the apparent and presumptive liability of those deep pockets, so as to maximize the magnitude of potential judgments and settlements. On the other hand, the pervasive inclusion of marginal defendants in most malpractice litigation contributes dramatically to the practice of defensive medicine by the vast majority of US health care providers. This almost certainly increases the cost and dissatisfaction for patients treated within that same HCI.

These case studies also illustrate the fragmentation of the US HCI, the potential for adverse events related to patient "handoffs" from one provider to another, the antagonistic relationship between hospitals and attending staff, and the sense of oppression experienced by many providers related to the arguably unreasonable inclusion of certain providers as defendants in legal actions alleging medical negligence.

\section{Conclusion and future directions}

In conclusion, despite documented success in reducing medical errors in both surgical anesthesia and the Veterans Health Administration Medication Delivery System, adverse events and medical errors continue to represent an indelible stain upon the practice, reputation, and success of the US HCI. In that regard, what may be required to successfully attack the unacceptably high severity and volume of medical errors is a locally directed and organized initiative sponsored by individual health care organizations that is coordinated, supported, and guided by state and federal governmental and nongovernmental agencies. With such a comprehensive approach, trained and designated personnel at or consulting with diverse health care organizations would undertake the following:

1. Detect and observe the manifestation of medical mistakes in identifiable patient groups at greatest risk, and comprehend their cognizable root causes;

2. Scrutinize, decipher, and distribute data to clinicians and interested parties;

3. Execute error diminution stratagems based upon reanalysis and restructuring of health care systems;

4. Where required, call upon health care experts for technical support and on-site surveys;

5. Evaluate the impact of newly implemented programs on patient safety. ${ }^{8}$

Factors that presently reduce the efficacy of medical error prevention programs include:

1. Extant performance improvement and continuous quality improvement programs most often do not specifically address the issue of medical errors;

2. Initiatives aimed at medical error reduction traditionally operate in isolation;

3. Infection control and employee safety initiatives typically receive low priority at health centers; 
4. Passive error reporting systems suffer from incomplete reporting and underreporting of identifiable medical errors;

5. Active reporting systems are expensive to implement and maintain. Furthermore, active reporting systems that hold individuals responsible understandably suffer from intentional underreporting of errors;

6. The new reduced working hours of house staff has resulted in the new dilemma of frequent patient handoffs to the new doctors coming on service. These handoffs have resulted in a new potential pitfall for errors that will need to be addressed in the upcoming years;

7. Despite the present mandate for electronic records so that medical information can be more easily shared by collaborating physicians and subspecialties, the varied types of electronic medical records and their inability to communicate, and the added Health Insurance Portability and Accountability Act of 1996 mandate causing electronic sharing of information to be potentially fraught with loss of privacy, doom this initiative to failure. ${ }^{8}$

The Quality Interagency Coordination Task Force has concluded that "systems designed to facilitate quality improvement through error reduction can generate effective, useful reporting if those individuals who report are assured of confidentiality, protected from legal liability resulting from the report, provided with timely feedback on the data from the system, and are not unduly burdened by the effort involved in reporting."

\section{Disclosure}

The authors report no conflicts of interest in this work.

\section{References}

1. Smith CM. Origin and uses of primum non nocere: above all, do no harm! J Clin Pharmacol. 2005;45(4):371-377.

2. Brennan TA, Leape LL, Laird NM, et al; for Harvard Medical Practice Study I. Incidence of adverse events and negligence in hospitalized patients: results of the Harvard Medical Practice Study I; 1991. Qual Saf Health Care. 2004;13(2):145-151; discussion 151-152.
3. Brennan TA, Leape LL, Laird NM, et al. Incidence of adverse events and negligence in hospitalized patients: results of the Harvard Medical Practice Study I. N Engl J Med. 1991;324(6):370-376.

4. National Research Council. To Err is Human: Building a Safer Health System. Washington (DC): The National Academies Press; 2000.

5. Thomas EJ, Studdert DM, Burstin HR, et al. Incidence and types of adverse events and negligent care in Utah and Colorado. Med Care. 2000;38(3):261-271.

6. National Research Council. Crossing the Quality Chasm: A New Health System for the 21st Century. Washington (DC): The National Academies Press; 2001.

7. McGlynn EA, Asch SM, Adams J, et al. The quality of health care delivered to adults in the United States. $N$ Engl J Med. 2003;348(26):2635-2645.

8. UuIC.gov [homepage on the Internet]. Washington (DC): Quality Interagency Coordination Task Force. Available from: http://www. quic.gov/. Accessed April 25, 2012.

9. Leape LL, Brennan TA, Laird N, et al. The nature of adverse events in hospitalized patients: results of the Harvard Medical Practice Study II. N Engl J Med. 1991;324(6):377-384.

10. Chassin MR. Is health care ready for Six Sigma quality? Milbank $Q$. 1998;76(4):565-591, 510.

11. Wilhite T. The definition and underlying theory of Six Sigma. Available from: http://www.ehow.com/facts_6920556_definition-underlyingtheory-six-sigma.html. Accessed April 25, 2012.

12. Quality Interagency Coordination Task Force (QuIC). Understanding medical errors [chapter on the Internet]. In: QuIC. Doing What Counts for Patient Safety: Federal Actions to Reduce Medical Errors and Their Impact. Washington (DC): QuIC; 2000. Available from: http://www. quic.gov/report/mederr4.htm. Accessed April 25, 2012.

13. US Food and Drug Administration (FDA). How does FDA monitor safety after drugs are approved and marketed? Silver Spring (MD): US FDA; updated May 23, 2012. Available from: http://www.fda.gov/ AboutFDA/Transparency/Basics/ucm305058.htm. Accessed May 30, 2012.

14. US Department of Health and Human Services, Food and Drug Administration. Guidance for industry oversight of clinical investigations: a risk-based approach to monitoring [draft guidance]. Silver Spring (MD): US FDA; August 24, 2011. Available from: http://www.fda. gov/downloads/Drugs/GuidanceComplianceRegulatoryInformation/ Guidances/UCM269919.pdf. Accessed May 30, 2012.

15. Emori TG, Culver DH, Horan TC, et al. National Nosocomial Infections Surveillance System (NNIS): description of surveillance methods. Am J Infect Control. 1991;19(1):19-35.

16. Leape LL. Reporting of adverse events. N Engl J Med. 2002;347(20): 1633-1638.

17. Orkin FW. Patient monitoring during anesthesia as an exercise in technology assessment. In: Saidman LJ, Smith NT, editors. Monitoring in Anesthesia. 3rd ed. London: Butterworth-Heinemann; 1993.

18. Findlay S, editor. Reducing Medical Errors and Improving Patient Safety: Success from the Front Lines of Medicine. Washington (DC): National Coalition on Health Care and Institute for Health Care Improvement; 2000.

International Journal of General Medicine

\section{Publish your work in this journal}

The International Journal of General Medicine is an international, peer-reviewed open-access journal that focuses on general and internal medicine, pathogenesis, epidemiology, diagnosis, monitoring and treatment protocols. The journal is characterized by the rapid reporting of reviews, original research and clinical studies across all disease areas.

A key focus is the elucidation of disease processes and management protocols resulting in improved outcomes for the patient.The manuscript management system is completely online and includes a very quick and fair peer-review system. Visit http://www.dovepress.com/ testimonials.php to read real quotes from published authors. 